Nora Derbal

Philanthropie in Saudi-Arabien 
Maecenata Schriften Band 9 
Nora Derbal

\section{Philanthropie in Saudi-Arabien}

Bestandsaufnahme und Untersuchung der organisierten wohltätigen Praxis in Djidda

2012 
Anschrift der Autorin:

Nora Derbal

Berlin Graduate School Muslim Cultures and Societies (BGSMCS)

Freie Universität Berlin

Altensteinstr. 48

14195 Berlin

derbal@bgsmcs.fu-berlin.de

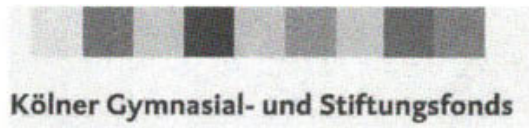

Kölner Gymnasial- und Stiftungsfonds
Die Publikation ist die überarbeitete und aktualisierte Magisterarbeit von Nora Derbal, eingereicht im April 2011 am Institut für Islamwissenschaft der Freien Universität Berlin, gefördert durch den Kölner Gymnasial und Stiftungsfonds.

Der 6-monatige Auslandsaufenthalt zum Studium in SaudiArabien, der die Grundlage der Feldforschung für diese Arbeit darstellt, wurde außerdem großzügig durch den Deutschen Akademischen Auslandsdienst (DAAD) gefördert.

\section{DAAD}

Bibliografische Information der Deutschen Nationalbibliothek

Die Deutsche Nationalbibliothek verzeichnet diese Publikation in der Deutschen Nationalbibliografie; detaillierte bibliografische Daten sind im Internet über http://dnb.d-nb. de abrufbar.

ISBN 978-3-8282-0564-2

C Lucius \& Lucius Verlagsgesellschaft mbH Stuttgart 2012

Gerokstraße $51 \cdot$ D-70184 Stuttgart

www.luciusverlag.com

Das Werk einschließlich aller seiner Teile ist urheberrechtlich geschützt. Jede Verwertung außerhalb der engen Grenzen des Urheberrechtsgesetzes ist ohne Zustimmung des Verlags unzulässig und strafbar. Das gilt insbesondere für Vervielfältigungen, Übersetzungen, Mikroverfilmungen und die Einspeicherung und Verarbeitung in elektronischen Systemen.

Umschlaggestaltung: I. Devaux, Stuttgart

Druck und Bindung: Rosch-Buch, Scheßlitz 